\title{
NECESIDADES DE INFORMACION EN LAS EMPRESAS: ESTUDIO DE UN CASO
}

\author{
E. Sanz", L. Rubio**
}

Resumen: El esiudio de las necesidades de información en las empresas es una tarea compleja y urgente que se está acometiendo desde distintas vertientes, con el fin de ir completando el conocimiento que se tiene de este tipo de usuario de información. En este sentido, en el trabajo se estudian las necesidades de información de las empresas norteamericanas del estado de Indiana, a partir de una serie de entrevistas con responsables de centros de información y servicios de desarrollo, que atendian las distintas demandas de las empresas. Asimismo, en el trabajo se ha utilizado una encuesta realizada por la Universidad del Estado de Indiana sobre el uso y los tipos de información más demandados por las empresas.

Palabras clave: Información para la empresa, necesidades de información, canales formales, canales informales, tipos de información.

Abstract: The study of information needs in companies is a complex and urgent task, undertaken in different ways, in order to have a good knowledge on this type of information user. In this way, the information needs of the American companies of the Indiana State are studied in this article, from several meetings with local librarians and persons in charge of different services which assist development of the local companies. In the study, a survey performed by the Indiana State University, which analyzed the information use and the types of information more frequently required by the companies was also used.

Keywords: Information for industries, information needs, formal channels, informal channels, types of information.

\section{Introducción}

Las empresas disponen de y utilizan una gran cantidad y variedad de recursos de información para resolver sus necesidades, ya que, en general, éstas son muy complejas y dinámicas, y dependen en muchos casos del tema específico en que estén trabajando en ese momento, de la toma de decisiones que tengan que realizar o de cualquier otra actividad empresarial que se esté llevando a cabo.

Los canales que las empresas utilizan para cubrir sus necesidades de información suelen ser una combinación de canales formales e informales. Estos últimos son hoy en día de gran trascendencia, puesto que cada vez es más utilizada la información de tipo efímero que no es sometida a contrastación y que requiere menos recursos para su gestión. En este sentido, son las pequeñas y medianas empresas las que tienen mayor dependencia de los canales informales, ya que carecen en su mayoría de departamento de documentación o biblioteca y resuelven sus problemas de información, en gran medida, mediante la utilización de este tipo

* Departamento de Biblioteconomía y Documentación, Universidad Carlos III de Madrid.

** Becaria del Ministerio de Educación para la convocatoria del Personal Investigador en la Universidad de Indiana.

Recibido: $18-9-92$. 
de recursos, que canalizan a través de los distintos departamentos. Mientras que los canales formales son utilizados preferentemente por las grandes empresas, donde es más frecuente encontrar departamento de documentación o biblioteca dentro de la estructura empresarial, y por lo tanto personal cualificado para realizar una gestión adecuada de la información.

A partir de las investigaciones llevadas a cabo por Allen (1) en la década de los 60 , se empezó a conocer el papel que jugaban los canales informales a la hora de satisfacer las necesidades de información en las organizaciones. Los resultados obtenidos permitieron demostrar el papel vital que este tipo de fuentes jugaba en las empresas, determinando en muchos casos su capacidad competitiva, y por tanto su propia existencia. Recientemente, las investigaciones realizadas por el Centre for Exploitation of Science and Technology y la British Library sobre los hábitos de información de las empresas británicas (2) han permitido demostrar que, lejos de desaparecer esa tendencia en la utilización de los canales informales, éstos son cada vez más usados y constituyen la fuente de información más valorada dentro de la empresa.

En cuanto a los tipos de canales informales utilizados con mayor frecuencia por las empresas para obtener información, son en general: los contactos personales, el teléfono, fax, y recientemente está siendo muy utilizado el correo electrónico para transferir volúmenes importantes de información dentro de la propia empresa, o con el exterior; concretamente la que se mantiene con colaboradores, suministradores o clientes. Este tipo de canales permite a la empresa disponer de una gran cantidad de información sobre su entorno de trabajo, y - lo que es más importante- - en un período muy corto de tiempo. Sin embargo, las empresas que basan su estrategia de información sólo en este tipo de canales están en franca desventaja frente a las que de forma paralela utilizan los canales formales para completar sus necesidades de información, puesto que en este tipo de recursos se cuenta con una gran experiencia que está permitiendo elaborar una amplia y diversificada gama de productos (3). Por tanto, no tener en cuenta este tipo de fuentes a la hora de planificar o desarrollar las diversas actividades de la empresa supone disminuir enormemente su adecuada capacidad de respuesta ante los continuos retos a que se ve sometida.

Entre los canales formales más demandados por las empresas para cubrir sus necesidades de información destacan, en primer lugar, las publicaciones periódicas, que son utilizadas preferentemente para recuperar documentación de tipo científico-técnico o con el fin de tener un conocimiento del entorno donde trabaja la empresa (2). Las patentes, actas de congresos, informes y monografías también tienen una demanda importante pero con una frecuencia muy inferior a la de las publicaciones periódicas $(4,5)$. Sin embargo las bases de datos contrariamente a lo que podía esperarse - no ocupan un papel importante en la solución de los problemas de información de las empresas. Esto puede deberse al gran desconocimiento que tienen de su temática o a la diversidad y complejidad que ha supuesto para un usuario no experimentado el acceso a este tipo de recurso (6).

En cuanto a los tipos de información más importantes para las empresas hay que mencionar los que se refieren a temáticas que afectan directamente a su actividad comercial y planificación estratégica, como estadística, que permite a las empresas tener un buen conocimiento de distintos aspectos de la población o del 
mercado donde desarrolla sus actividades. En este sentido, este tipo de fuentes se encuentra muy diversificado, y comprende desde estadística demográfica, que contiene información detallada sobre diferentes características de la población, como datos de empleo, educación, características familiares, etc., que hacen de esta información una herramienta imprescindible para realizar una adecuada segmentación del mercado, hasta estadística de sectores industriales y productos, que proporciona una valiosa información sobre la estructura industrial, y sus actividades productivas; sin olvidar la información estadística del mercado laboral, que permite a las empresas conocer las tendencias de dicho mercado, salarios, condiciones de trabajo, etc. (7).

Otro tipo de información importante para las empresas son las normas, puesto que cualquier empresa con actividad exportadora necesita conocer la normativa vigente en los países donde pretenda vender sus productos. La información sobre mercados y empresas es también muy útil y apreciada, pues permite a las empresas conocer a sus competidores y la clase de productos que éstos elaboran $(8,9)$.

La información sobre financiación proporciona a la empresa datos sobre cómo obtener recursos económicos a partir de programas promovidos por las distintas administraciones o entidades financieras (10). Esta información es fundamental para las pequeñas empresas, debido a que sus presupuestos son extremadamente ajustados y cualquier gasto destinado a modernizar sus procesos productivos o sus actividades de promoción o de marketing supone serios problemas de tesorería. Por tanto, la información sobre programas de ayudas, subvenciones o créditos es sumamente importante para las empresas y en particular para las pequeñas.

Las oportunidades de negocio y prospecciones internacionales constituyen otro tipo de información muy utilizada en el mundo empresarial, pues, en una situación como la actual, donde los mercados están globalizados, las empresas necesitan conocer qué compañías en el mismo o en otro país son las más apropiadas para realizar algún tipo de cooperación (capital-riesgo, distribución, subcontratación, etc.).

La información cientifica y técnica es otro tipo de recurso importante para las empresas, pues cada vez la competencia es mayor y en muchos casos su supervivencia dependerá de una adecuada y continua adaptación de sus procesos productivos a las demandas de un mercado cada vez más dinámico y flexible (11).

\section{Estudio de las necesidades y uso de la información en las empresas americanas}

El estudio fue realizado con el fin de conocer los hábitos y necesidades de información de las empresas americanas, y concretamente las situadas en el estado de Indiana con motivo de la estancia de los autores en la School of Library and Information Science de la Universidad de Indiana. El tejido industrial de este estado es muy similar al de determinadas regiones españolas, con una importante concentración de empresas en sectores tradicionales, como los derivados de las actividades agrarias y ganaderas, conviviendo con un núcleo cada vez más importante de empresas que trabajan en sectores de tecnologías punta, como son el electrónico e informático. 
Los objetivos del estudio fueron los siguientes:

a) Identificar la variedad de fuentes de información utilizadas por las empresas, así como establecer el uso de los canales formales e informales como medio de cubrir sus necesidades de información.

b) Determinar las funciones de un departamento de información dentro de la empresa, y su papel en la gestión de información.

c) Determinar las relaciones existentes entre la empresa y los centros de investigación tanto públicos como privados, con el fin de conocer la transferencia de tecnología que se estaba produciendo.

\subsection{Metodología}

La metodología seguida en el estudio implicó una serie de entrevistas con especialistas de información que atendían las demandas documentales de las empresas, y con directores de diferentes servicios de la Universidad de Indiana que tenian como objetivo el fomentar el desarrollo de las empresas locales. Las entrevistas se realizaron a partir de un cuestionario elaborado previamente y que constaba de preguntas abiertas, con el fin de que el entrevistado pudiera dar su opinión libremente, y al mismo tiempo recoger las respuestas en un marco homogéneo que nos permitiera agrupar los resultados. En el estudio también se utilizó una bibliografia específica y, en particular, una encuesta realizada por el Center for Research and Management Service de la Universidad del Estado de Indiana (12), donde se recogían datos sobre el uso de las bibliotecas y el tipo de información más utilizada por 500 empresas de dicho estado.

\subsection{Resultados}

Los resultados sobre el uso y las necesidades de información de las empresas que se obtuvieron en el estudio se describen a continuación:

\subsubsection{Relaciones con otras empresas y centros de investigación universitarios}

Los contactos entre la empresa y la universidad eran frecuentes en aquellos casos en que las empresas estaban trabajando en tecnologías punta, o cuando se trataba de grandes empresas. Sin embargo, en otros casos, por ejemplo, cuando se trataba de empresas pequeñas o medianas, o cuando sus actividades se enmarcaban en campos tradicionales, no solían mantener ningún tipo de relación con la universidad, y en muchos casos esto era debido a que la empresa sentía que su nivel tecnológico era demasiado bajo para que fuese interesante establecer algún tipo de colaboración con la universidad.

En cuanto a la transferencia de tecnología, se demostró que había un flujo importante entre empresas que trabajan en sectores avanzados y la universidad. En este sentido, el estado de Indiana cuenta con un centro de transferencia de tecnología que trata de fomentar en las empresas el uso de la investigación que 
realizan los departamentos universitarios, y para ello se ha marcado los siguientes objetivos: estimular la transferencia y comercializar los derechos de la propiedad intelectual, facilitar el desarrollo de la colaboración industrial, y proveer de recursos y asistencia a las empresas por parte de profesores y estudiantes.

Otra característica que presentaron las empresas fue su escasa predisposición a mantener relaciones con empresas de fuera. La gestión empresarial tenía una visión excesivamente localista, y no manifestaba mucho interés por conocer empresas extranjeras para establecer alguna clase de colaboración. En este sentido, la encuesta llevada a cabo por el Center for Research and Management Service (12) mostró que sólo un $7 \%$ de las empresas de Indiana estaban interesadas en obtener información sobre exportación.

\subsubsection{Fuentes de información utilizadas por las empresas}

La información interna, elaborada a partir del conocimiento que la empresa ha ido acumulando en el desarrollo de sus actividades, fue considerada muy importante y valiosa, y concretamente era utilizada en actividades fundamentales en el desarrollo de la empresa, por ejemplo, en la toma de decisiones.

La información externa, elaborada en el exterior de la compañía y disponible a partir de canales formales o informales, también fue considerada de gran valor por las empresas, principalmente para cubrir las necesidades de información relativas a investigación de mercados, nuevas tecnologías, o legislación. En general, se comprobó que la pequeña y mediana empresa desconocía la mayoría de los recursos de información que tenían disponibles los centros de información; por esta razón, en muchos casos sólo usaban una escasa variedad de ellos.

La información externa más importante para la empresa la obtenían a partir de canales informales, que eran usados por la mayoría de ellas de una forma regular. Casi la mitad de las empresas que pedian información comercial o de negocios manifestaron la necesidad de recibir esta información en menos de dos dias, y el acceso mediante ordenador a través de la línea telefónica fue seleccionado como el método más interesante para obtener esta información por un porcentaje similar de empresas.

En cuanto a los canales informales, los más utilizados fueron: el teléfono, fax, contactos personales, y correo electrónico. Esta última fuente demostró tener un importante desarrollo entre las empresas, como medio de intercambiar información dentro o fuera de las mismas.

Como se ha comentado anteriormente, los canales formales eran en muchos casos muy desconocidos por las empresas, principalmente las pequeñas y medianas. Sin embargo, cada dia es mayor el interés que manifiestan por utilizar este tipo de recursos. En este sentido, las fuentes más utilizadas por las empresas fueron:

- Las revistas comerciales; en general este recurso era muy utilizado porque permitía a las empresas obtener una completa información del sector donde desarrollaban sus actividades.

-. Las fuentes estadísticas también demostraron ser muy demandadas por las empresas, y principalmente por las pequeñas y medianas, que para acceder 
a este tipo de recursos tenían que recurrir a servicios de información externos a la propia empresa, puesto que en la mayoría de los casos carecían de biblioteca o centro de documentación, y cuando lo tenían no contaba con una variedad importante de fondos.

- Las patentes eran usadas por las empresas para obtener información sobre desarrollos técnicos, y fueron las empresas que trabajaban en los sectores de la química o de las comunicaciones las que más demandaban este tipo de recurso.

- Las bases de datos eran utilizadas, generalmente, sólo cuando la empresa necesitaba hacer una puesta al día sobre la información más novedosa en su campo de trabajo.

- Los informes gubernamentales fueron muy utilizados por las empresas grandes, mientras que la medianas y pequeñas no demostraron mucho interés por este tipo de recurso, posiblemente por ser muy desconocido para ellas.

- Las normas fueron menos demandadas por las empresas, debido a que, como se ha comentado anteriormente, éstas demostraron unos objetivos excesivamente locales.

\subsubsection{Tipos de información demandados por las empresas}

Las empresas (pequeñas, medianas y grandes) necesitaban en primer lugar información de tipo comercial y financiero. La información sobre condiciones económicas demostró ser de gran interés para las pequeñas y medianas empresas. Estos datos difieren de forma importante de los obtenidos en empresas españolas (11), para las cuales la información científico-técnica es la que tiene mayor interés.

Otros tipos de información muy solicitados por las empresas fueron los relacionados con el desarrollo de nuevos productos, sobre nuevas tecnologías, ventas, competidores, formación de empleados, producción, y nuevos mercados. Sin embargo, la información sobre exportación, relaciones empresariales y distribución tuvo escaso interés para las empresas estudiadas.

\subsubsection{Funciones especificas del departamento de información en la empresa}

La mayoría de las pequeñas empresas no disponían de departamento de información o biblioteca, de tal manera que resolvían sus problemas de información utilizando los servicios de las bibliotecas públicas, universitarias, o de las asociaciones profesionales.

Era frecuente que las empresas medianas y, sobre todo, las grandes, tuvieran departamento de documentación o biblioteca; sin embargo, debido a la enorme variedad de tipos de información que necesitaban, también recurrian a otros centros de información con el fin de complementar sus recursos documentales.

En cuanto a las funciones que debía desarrollar el departamento de documentación dentro de la empresa, la más importante, según los entrevistados, era tener un buen conocimiento del medio donde estaba trabajando la empresa (competido- 
res, suministradores, clientes, etc.) con el fin de trasladar esta información a otros departamentos e incidir en sus actividades, adecuándolas a las necesidades detectadas en el entorno.

Otras funciones del departamento de información estaban ligadas a concepciones más tradicionales, como eran: la de obtener y suministrar la información solicitada, conocer los distintos recursos disponibles para la empresa y elaborar informes a medida sobre temas específicos en los que se estuviera trabajando.

\subsubsection{Otros centros de información utilizados por las empresas}

Según la encuesta que la Universidad del Estado de Indiana realizó a las empresas (12), sólo el $9 \%$ de ellas utilizaba exclusivamente su propio departamento de documentación o biblioteca para cubrir sus necesidades de información, generalmente la de tipo comercial y de negocios, y en la mayoría de los casos estas empresas completaban sus demandas documentales utilizando los servicios de información de otros centros. La elección de éstos dependía del tipo de información que necesitaran. En este sentido, los centros más utilizados por las empresas fueron: las bibliotecas públicas, las universitarias, las asociaciones profesionales, las cámaras de comercio, las agencias estatales y federales, las organizaciones de desarrollo económico, y las empresas consultoras.

\section{Conclusiones}

Hay varias conclusiones que pueden ser sacadas a partir de este estudio, que son extrapolables a la situación en España, pues las empresas españolas presentan una problemática muy similar a las estudiadas, en cuanto a sus necesidades de información.

La primera conclusión es que las empresas necesitan utilizar una gran cantidad y variedad de fuentes de información para realizar un adecuado desarrollo de sus actividades. Sin embargo, en la mayoría de los casos no lo hacen porque desconocen los recursos de información que tienen a su alcance en las bibliotecas y otros centros de información. Esta situación se evitaría si estos centros dieran a conocer a las empresas, mediante folletos, boletines, o cualquier otro medio informativo, los servicios disponibles, los tipos de información que componen sus fondos, la forma de acceder a ellos, y la relación de precios; asimismo es fundamental actualizar periódicamente esta información con las nuevas adquisiciones.

La segunda conclusión es que las empresas necesitan tener en su estructura un departamento de documentación y personal con una formación adecuada, que maneje todos los recursos de información tanto formales como informales, y que a partir de ellos sea capaz de elaborar productos de información con alto valor añadido.

La tercera y última conclusión es que la empresa necesita conocer los procesos de investigación que se están llevando a cabo en las universidades y otros centros de investigación, con el fin de utilizarlos para mejorar sus actividades productivas. 


\section{Agradecimientos}

La realización de este estudio fue posible gracias a las ayudas económicas de la Comunidad de Madrid y de la Comisión Interministerial de Ciencia y Tecnología (CICYT). También queremos agradecer la colaboración prestada por el profesorado de la School of Library and Information Science de la Universidad de Indiana, en cuyo departamento se realizó este trabajo, y especialmente la de su decano, profesor Blaise Cronin.

También queremos extender nuestro agradecimiento a Jay Marme, Judith Copler, Susan Jackson y Carol Roger por sus comentarios y sugerencias a diferentes aspectos de este trabajo.

\section{Bibliografia}

1. ALLEN, T. J. Communications in the research and development laboratory, Technology Review, 70 (1), 31-37, 1967.

2. CENTRE FOR EXPLOITATION OF SCIENCE AND TECHNOLOGY, BRITISH LIBRARY DOCUMENT SUPPLY CENTRE. Information flows into industrial research, Londres: British Library Board, 1989.

3. KAYE, D. Information and business. An introduction. London: Library Association Publishing Ltd, 1991.

4. SANZ, E.; HERNANDEZ, A.; ARAGON, I.; ALVAREZ, C. Study of scientific information needs of Spanish industry. 10ème Congres IDT, París, 22-24, June 1993.

5. WEEDMAN, J. Informal and formal channels in boundary-spanning communication, Journal of the American Society for Information Science, 43 (3), 257-267, 1992.

6. DRENTH, H.; TSENG, G.; MORRIS, A. Tracking British and European online business information: A guide to the guides, Online Review, 15 (6), 355-365, 1991.

7. MORT, D. Statistical sources for business, Aslib Information, 19 (4), 123-125, 1991.

8. WOOD, T. European business information sources, Aslib Information, 19 (4), 128-132, 1991.

9. OWEN, T. Getting down to basics, Aslib Information, 19 (4), 116-118, 1991.

10. WILSON, T. Information for business: The business of information, Aslib Proceedings, 39 (10), 275-279, 1987.

11. SANZ, E. Servicios de información para las empresas del Sector de Electrónica e Informática, Jornadas Internacionales sobre Información Electrónica y Empresa, San Sebastián, 1990.

12. CHIESL, N. State of Indiana. Library study 1990, Indiana State University, Center for Research and Management Services, 1990. 